\title{
InterCriteria Analysis of a Model Parameters Identification using Genetic Algorithm
}

\author{
Olympia Roeva \\ Institute of Biophysics and Biomedical Engineering \\ Bulgarian Academy of Science \\ Sofia, Bulgaria \\ E-mail: olympia@ biomed.bas.bg \\ Peter Vassilev \\ Institute of Biophysics and Biomedical Engineering \\ Bulgarian Academy of Science \\ Sofia, Bulgaria \\ E-mail: peter.vassilev@gmail.com
}

\author{
Stefka Fidanova \\ Institute of Information and Communication Technology \\ Bulgarian Academy of Science \\ Sofia, Bulgaria \\ E-mail: stefka@parallel.bas.bg \\ Pawel Gepner \\ Intel Corporation, Pipers Way \\ Swindon Wiltshire SN3 1RJ \\ United Kingdom \\ E-mail: pawel.gepner@intel.com
}

\begin{abstract}
In this paper we apply an approach based on the apparatus of the Index Matrices and the Intuitionistic Fuzzy Sets - namely InterCriteria Analysis. The main idea is to use the InterCriteria Analysis to establish the existing relations and dependencies of defined parameters in non-linear model of an E. coli fed-batch cultivation process. Moreover, based on results of series of identification procedures we observe the mutual relations between model parameters and considered optimization techniques outcomes, such as execution time and objective function value. Based on InterCriteria Analysis we examine the obtained identification results and discuss the conclusions about existing relations and dependencies between defined, in terms of InterCriteria Analysis, criteria.
\end{abstract}

Index Terms - InterCriteria Analysis; Index matrices; Intuitionistic Fuzzy Sets; Genetic Algorithm; chromosomes; parameter identification; E. coli; fed-batch cultivation process.

\section{INTRODUCTION}

$\mathbf{T}$ HE InterCriteria Analysis (ICA) is developed with the aim to gain additional insight into the nature of the criteria involved and discover on this basis existing relations between the criteria themselves [8]. It is based on the apparatus of the Index Matrices (IM) [10], [11], and the Intuitionistic Fuzzy Sets [12] and can be applied for decision making in different areas of science and practice. The approach has been discussed in a several papers [10], [14], [15], [16]. In [8] a possibility of the ICA method for criterion value prediction, proposing two algorithms, is presented. In [16] a discussion on the threshold values in the ICA was further elaborated. But, up to now, considering ICA application, the only applications reported are in one area: namely, EU member states competitiveness analysis [14], [15]. Encouraging results of these first applications of the ICA provoke us to use the method for establishing and identifying the relations between parameters of the mathematical model of an E. coli fedbatch cultivation process. The model parameters are further considered as criteria in terms of ICA.
In the case of modelling of cultivation processes ICA approach could be very useful. Cultivation processes are characterized with complex, non-linear dynamic and their modelling is a hard combinatorial optimization problem. On the one hand, the parameter identification is of key importance for modelling process and additional knowledge about the model parameters relations will be extremely useful to improve the model accuracy. On the other hand, the information may be used to improve the performance of the used optimization algorithms if, for instance, some algorithm outcomes are added to the considered criteria. Thus, the relations between model parameters and optimization algorithm performance will be established.

In this paper we applied the ICA to establish the basic relations between the parameters in the model of an $E$. coli fedbatch cultivation process. The existing relations are identified based on results of a series of parameters identification procedures. The use of meta-heuristic techniques such as Genetic Algorithms (GAs) has received more and more attention [3]. These methods offer good solutions, even global optima, within reasonable computing time [17], so we choose to use genetic algorithms for estimation of the model parameters.

The paper is organized as follows. The background of InterCriteria Analysis is given in Section 2. The problem formulation is described in Section 3. The numerical results and a discussion are presented in Section 4. Conclusion remarks are done in Section 5.

\section{INTERCRITERIA ANALYSIS}

Here we expand on the idea proposed in [8]. Following [8] and [12] we will obtain an Intuitionistic Fuzzy Pair (IFP) as the degrees of "agreement" and "disagreement" between two criteria applied on different objects. We remind briefly that an IFP is an ordered pair of real non-negative numbers $\langle a, b\rangle$ such that:

$$
a+b \leq 1
$$


For clarity, let us be given an IM (see [10]) whose index sets consist of the names of the criteria (for rows) and objects (for columns). The elements of this IM are further supposed to be real numbers (in the general case, this is not required). We will obtain an IM with index sets consisting of the names of the criteria (for rows and for columns) with elements IFPs corresponding to the "agreement" and "disagreement" of the respective criteria.

Two things are further supposed (which are not always guaranteed in practice and, when not fulfilled, present an interesting direction for new research in themselves):

1) All criteria provide an evaluation for all objects (i.e. there are no inapplicable criteria for a given object) and all these evaluations are available (no missing evaluations).

2) All the evaluations of a given criteria can be compared amongst themselves.

Further by $O$ we denote the set of all objects $O_{1}, O_{2}, \ldots, O_{n}$ being evaluated, and by $C(O)$ the set of values assigned by a given criteria $C$ to the objects, i.e.

$$
\begin{aligned}
& O \stackrel{\text { def }}{=}\left\{O_{1}, O_{2}, \ldots, O_{n}\right\}, \\
& C(O) \stackrel{\text { def }}{=}\left\{C\left(O_{1}\right), C\left(O_{2}\right), \ldots, C\left(O_{n}\right)\right\} .
\end{aligned}
$$

Let:

$$
C^{*}(O) \stackrel{\text { def }}{=}\{\langle x, y\rangle \mid x \neq y \&\langle x, y\rangle \in C(O) \times C(O)\} .
$$

In order to compare two criteria we must construct the vector of all internal comparisons of each criteria, which fulfill exactly one of three relations $R, \bar{R}$ and $\tilde{R}$. In other words, we require that for a fixed criterion $C$ and any ordered pair $\langle x, y\rangle \in C^{*}(O)$ it is true:

$$
\begin{aligned}
& \langle x, y\rangle \in R \Leftrightarrow\langle y, x\rangle \in \bar{R}, \\
& \langle x, y\rangle \in \tilde{R} \Leftrightarrow\langle x, y\rangle \notin(R \cup \bar{R}), \\
& R \cup \bar{R} \cup \tilde{R}=C^{*}(O) .
\end{aligned}
$$

From the above it is seen that we need only consider a subset of $C(O) \times C(O)$ for the effective calculation of the vector of internal comparisons (denoted further by $V(C)$ ) since from $(1),(2)$ and (3) it follows that if we know what is the relation between $x$ and $y$ we also know what is the relation between $y$ and $x$. Thus we will only consider lexicographically ordered pairs $\langle x, y\rangle$. Let, for brevity:

$$
C_{i, j}=\left\langle C\left(O_{i}\right), C\left(O_{j}\right)\right\rangle .
$$

Then for a fixed criterion $C$ we construct the vector:

$$
\begin{array}{r}
V(C)=\left\{C_{1,2}, C_{1,3}, \ldots, C_{1, n}, C_{2,3}, C_{2,4}, \ldots\right. \\
\left.C_{2, n}, C_{3,4}, \ldots, C_{3, n}, \ldots, C_{n-1, n}\right\}
\end{array}
$$

It can be easily seen that it has exactly $\frac{n(n-1)}{2}$ elements. Further, to simplify our considerations, we replace the vector
$V(C)$ with $\hat{V}(C)$, where for each $1 \leq k \leq \frac{n(n-1)}{2}$ for the $k$-th component it is true:

$$
\hat{V}_{k}(C)=\left\{\begin{aligned}
1 & \text { iff } V_{k}(C) \in R \\
-1 & \text { iff } V_{k}(C) \in \bar{R} \\
0 & \text { otherwise }
\end{aligned}\right.
$$

Then when comparing two criteria we determine the "degree of agreement" between the two as the number of matching components (divided by the length of the vector for normalization purposes). This can be done in several ways, e.g. by counting the matches or by taking the complement of the Hamming distance. The "degree of disagreement" is the number of components of opposing signs in the two vectors (again normalized by the length). This also may be done in various ways. A pseudocode of the algorithm used in this study for calculating the degrees of agreement and disagreement between two criteria $C$ and $C^{\prime}$ is presented below.

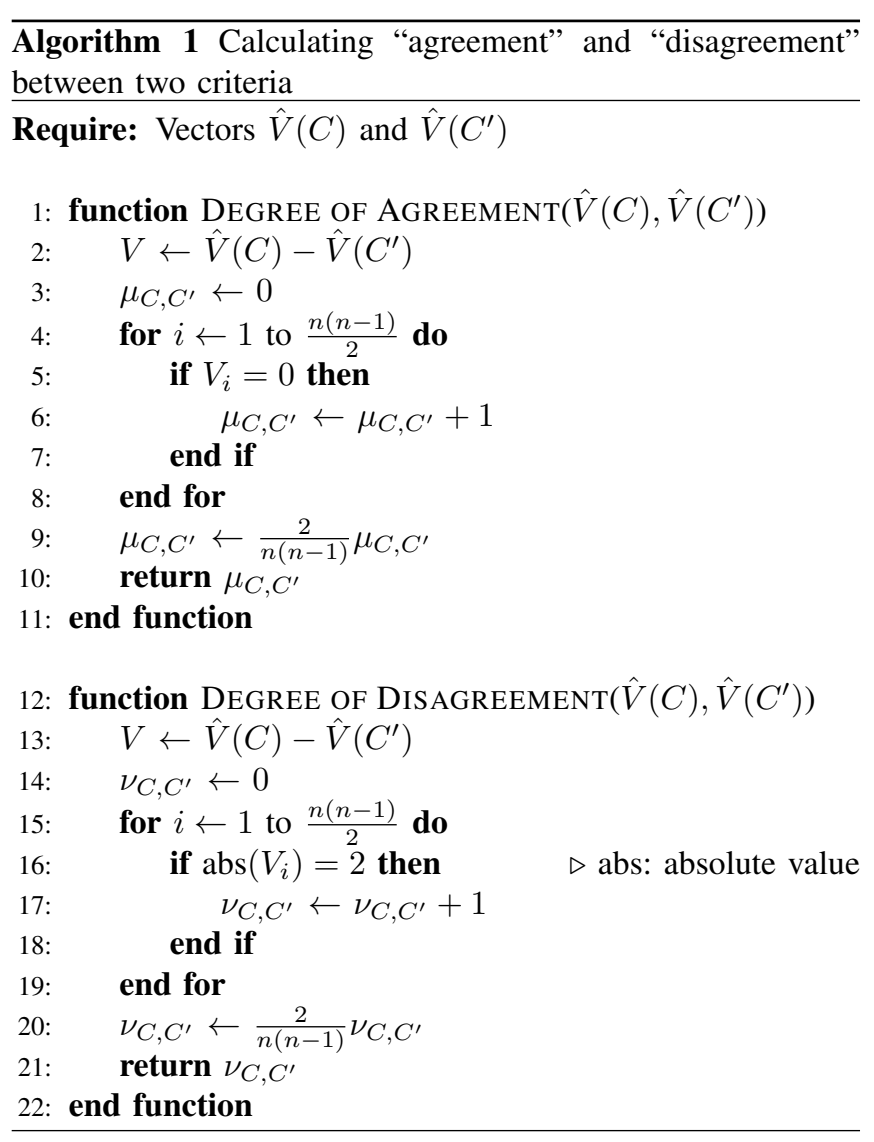

It is obvious (from the way of calculation) that for $\mu_{C, C^{\prime}}$, $\nu_{C, C^{\prime}}$, we have:

$$
\mu_{C, C^{\prime}}=\mu_{C^{\prime}, C}, \nu_{C, C^{\prime}}=\nu_{C^{\prime}, C} .
$$

Also, $\left\langle\mu_{C, C^{\prime}}, \nu_{C, C^{\prime}}\right\rangle$ is an IFP. 


\section{PRoblem Formulation}

Let us use the following non-linear differential equation system to describe the $E$. coli fed-batch cultivation process [1], [4]:

$$
\begin{gathered}
\frac{d X}{d t}=\mu X-\frac{F_{i n}}{V} X \\
\frac{d S}{d t}=-q_{S} X+\frac{F_{i n}}{V}\left(S_{i n}-S\right), \\
\frac{d V}{d t}=F_{i n}
\end{gathered}
$$

where

$$
\mu=\mu_{\max } \frac{S}{k_{S}+S}, \quad q_{S}=\frac{1}{Y_{S / X}} \mu
$$

and $X$ is the biomass concentration, $[\mathrm{g} / \mathrm{l}] ; S$ is the substrate concentration, $[\mathrm{g} / \mathrm{l}] ; F_{\text {in }}$ is the feeding rate, $[1 / \mathrm{h}] ; V$ is the bioreactor volume, [1]; $S_{i n}$ is the substrate concentration in the feeding solution, $[\mathrm{g} / \mathrm{l}] ; \mu$ and $q_{S}$ are the specific rate functions, $[1 / \mathrm{h}] ; \mu_{\max }$ is the maximum value of the $\mu,[1 / \mathrm{h}] ; k_{S}$ is the saturation constant, [g/l]; $Y_{S / X}$ is the yield coefficient, [-].

For the model (Eq. (4)-Eq. (7)) the parameters that will be identified are $\mu_{\max }, k_{S}$ and $Y_{S / X}$.

Let $Z_{\text {mod }} \stackrel{\text { def }}{=}\left[X_{\text {mod }} S_{\text {mod }}\right]$ (model predictions for biomass and substrate) and $Z_{\exp } \stackrel{\text { def }}{=}\left[X_{\exp } S_{\exp }\right]$ (known experimental data for biomass and substrate). Then putting $Z=Z_{\bmod }-Z_{\exp }$, we define the objective function as:

$$
J=\|Z\|^{2} \rightarrow \min ,
$$

where \|\| denotes the $\ell^{2}$-vector norm.

For the model parameters identification we use experimental data for biomass and glucose concentration of an E. coli MC4110 fed-batch fermentation process. The detailed description of the process condition and experimental data are presented in [2].

To estimate the model parameters we applied consistently 14 differently tuned GA. We use various population sizes from 5 to 200 chromosomes in the population. The number of generations is fixed to 200. The main GA operators and parameters are summarized in Table I. Because of the stochastic nature of the applied algorithms we perform series of 30 runs for each population size. Thus, we obtain the average, best and worst estimate of the parameters, as well as of the algorithm execution time and value of objective function. The detailed description of identification procedure is given in [7].

To perform ICA three IMs are constructed - the IM $A_{1}$ (Eq. 9) with the obtained average results, the IM $A_{2}$ (Eq. 10) with the best obtained results and IM $A_{3}$ (Eq. 11) with the worst obtained results. In addition to the presented in [7] results here the average, worst and best estimates for the tree model parameters in all 14 cases are given too. Thus, five criteria are considered $-C_{1}$ is parameter $\mu_{\max }, C_{2}$ is parameter $k_{S}, C_{3}$ is parameter $Y_{S / X}, C_{4}$ is objective function value $J$ and $C_{5}$ is resulting execution time $T$.
TABLE I

MAIN GA OPERATORS AND PARAMETERS

\begin{tabular}{|l|c|}
\hline Operator & Type \\
\hline fitness function & linear ranking \\
\hline selection function & roulette wheel selection \\
\hline crossover function & simple crossover \\
\hline mutation function & binary mutation \\
\hline reinsertion & fitness-based \\
\hline Parameter & Value \\
\hline generation gap & 0.97 \\
\hline crossover probability & 0.75 \\
\hline mutation probability & 0.01 \\
\hline number of generations & 200 \\
\hline
\end{tabular}

\section{NumericAl Results AND Discussion}

Computer specification to run all identification procedures are Intel Core i5-2329 3.0 GHz, 8 GB Memory, Windows 7 (64bit) operating system.

Based on the presented Algorithm 1 the ICA is implemented in the Matlab 7.5 environment. We obtain IMs that determine the degrees of "agreement" $\left(\mu_{C, C^{\prime}}\right)$ and "disagreement" $\left(\nu_{C, C^{\prime}}\right)$ between criteria for the three cases.

1) Case of average results:

Resulting degrees of "agreement" $\left(\mu_{C, C^{\prime}}\right)$ are as follows:

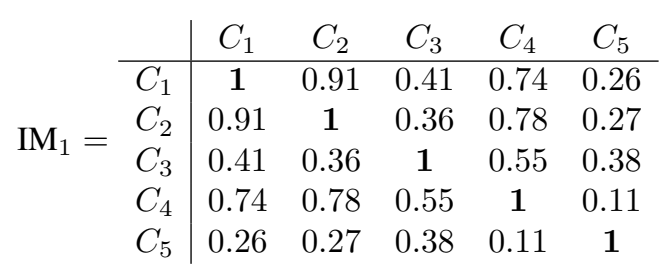

Resulting degrees of "disagreement" $\left(\nu_{C, C^{\prime}}\right)$ are as follows:

$$
\begin{array}{c|ccccc} 
& C_{1} & C_{2} & C_{3} & C_{4} & C_{5} \\
\hline \mathrm{IM}_{2}= & \mathbf{0} & 0.08 & 0.58 & 0.26 & 0.74 \\
C_{2} & 0.08 & \mathbf{0} & 0.62 & 0.21 & 0.71 \\
C_{3} & 0.58 & 0.62 & \mathbf{0} & 0.44 & 0.60 \\
C_{4} & 0.26 & 0.21 & 0.44 & \mathbf{0} & 0.89 \\
C_{5} & 0.74 & 0.71 & 0.60 & 0.89 & \mathbf{0}
\end{array}
$$

2) Case of worst results:

Resulting degrees of "agreement" $\left(\mu_{C, C^{\prime}}\right)$ are as follows:

$$
\begin{array}{c|ccccc} 
& C_{1} & C_{2} & C_{3} & C_{4} & C_{5} \\
\hline \mathrm{IM}_{5}= & \mathbf{1} & 0.79 & 0.34 & 0.88 & 0.14 \\
C_{2} & 0.79 & \mathbf{1} & 0.18 & 0.84 & 0.22 \\
C_{3} & 0.34 & 0.18 & \mathbf{1} & 0.33 & 0.64 \\
C_{4} & 0.88 & 0.84 & 0.33 & \mathbf{1} & 0.07 \\
C_{5} & 0.14 & 0.22 & 0.64 & 0.07 & \mathbf{1}
\end{array}
$$

Resulting degrees of "disagreement" $\left(\nu_{C, C^{\prime}}\right)$ are as follows: 
$A_{1}($ average $)=$

\begin{tabular}{l|cccccccccccccc} 
& $\mathrm{GA}_{5}$ & $\mathrm{GA}_{10}$ & $\mathrm{GA}_{20}$ & $\mathrm{GA}_{30}$ & $\mathrm{GA}_{40}$ & $\mathrm{GA}_{50}$ & $\mathrm{GA}_{60}$ & $\mathrm{GA}_{70}$ & $\mathrm{GA}_{80}$ & $\mathrm{GA}_{90}$ & $\mathrm{GA}_{100}$ & $\mathrm{GA}_{110}$ & $\mathrm{GA}_{150}$ & $\mathrm{GA}_{200}$ \\
\hline$C_{1}$ & 0.552 & 0.525 & 0.515 & 0.491 & 0.486 & 0.508 & 0.497 & 0.498 & 0.498 & 0.496 & 0.494 & 0.500 & 0.492 & 0.489 \\
$C_{2}$ & 0.022 & 0.021 & 0.018 & 0.013 & 0.010 & 0.016 & 0.014 & 0.013 & 0.014 & 0.014 & 0.012 & 0.015 & 0.013 & 0.011 \\
$C_{3}$ & 2.032 & 2.025 & 2.019 & 2.023 & 2.023 & 2.020 & 2.022 & 2.022 & 2.022 & 2.021 & 2.023 & 2.021 & 2.021 & 2.023 \\
$C_{4}$ & 6.271 & 5.838 & 4.760 & 4.561 & 4.646 & 4.607 & 4.580 & 4.568 & 4.578 & 4.570 & 4.553 & 4.547 & 4.560 & 4.545 \\
$C_{5}$ & 4.649 & 6.053 & 7.472 & 11.248 & 12.917 & 14.649 & 16.973 & 19.719 & 21.793 & 24.196 & 26.848 & 29.515 & 39.406 & 51.917
\end{tabular}

$$
A_{2}(\text { best })=
$$

\begin{tabular}{l|cccccccccccccc} 
& $\mathrm{GA}_{5}$ & $\mathrm{GA}_{10}$ & $\mathrm{GA}_{20}$ & $\mathrm{GA}_{30}$ & $\mathrm{GA}_{40}$ & $\mathrm{GA}_{50}$ & $\mathrm{GA}_{60}$ & $\mathrm{GA}_{70}$ & $\mathrm{GA}_{80}$ & $\mathrm{GA}_{90}$ & $\mathrm{GA}_{100}$ & $\mathrm{GA}_{110}$ & $\mathrm{GA}_{150}$ & $\mathrm{GA}_{200}$ \\
\hline$C_{1}$ & 0.491 & 0.480 & 0.494 & 0.491 & 0.488 & 0.492 & 0.488 & 0.490 & 0.484 & 0.491 & 0.488 & 0.486 & 0.488 & 0.488 \\
$C_{2}$ & 0.013 & 0.011 & 0.013 & 0.012 & 0.012 & 0.012 & 0.012 & 0.013 & 0.011 & 0.013 & 0.012 & 0.012 & 0.012 & 0.012 \\
$C_{3}$ & 2.023 & 2.024 & 2.018 & 2.023 & 2.020 & 2.023 & 2.020 & 2.019 & 2.019 & 2.020 & 2.019 & 2.021 & 2.019 & 2.018 \\
$C_{4}$ & 4.833 & 4.855 & 4.475 & 4.482 & 4.444 & 4.449 & 4.463 & 4.438 & 4.447 & 4.450 & 4.425 & 4.433 & 4.458 & 4.436 \\
$C_{5}$ & 4.867 & 5.912 & 7.675 & 11.295 & 13.229 & 15.007 & 17.316 & 20.062 & 22.667 & 24.757 & 26.926 & 30.015 & 39.780 & 52.323
\end{tabular}

$$
A_{3}(\text { worst })=
$$

\begin{tabular}{l|cccccccccccccc} 
& $\mathrm{GA}_{5}$ & $\mathrm{GA}_{10}$ & $\mathrm{GA}_{20}$ & $\mathrm{GA}_{30}$ & $\mathrm{GA}_{40}$ & $\mathrm{GA}_{50}$ & $\mathrm{GA}_{60}$ & $\mathrm{GA}_{70}$ & $\mathrm{GA}_{80}$ & $\mathrm{GA}_{90}$ & $\mathrm{GA}_{100}$ & $\mathrm{GA}_{110}$ & $\mathrm{GA}_{150}$ & $\mathrm{GA}_{200}$ \\
\hline$C_{1}$ & 0.577 & 0.538 & 0.544 & 0.518 & 0.521 & 0.517 & 0.515 & 0.510 & 0.510 & 0.505 & 0.489 & 0.510 & 0.504 & 0.511 \\
$C_{2}$ & 0.015 & 0.026 & 0.024 & 0.018 & 0.019 & 0.018 & 0.018 & 0.016 & 0.017 & 0.015 & 0.012 & 0.016 & 0.015 & 0.016 \\
$C_{3}$ & 2.037 & 1.995 & 2.019 & 2.021 & 2.021 & 2.021 & 2.020 & 2.022 & 2.021 & 2.022 & 2.022 & 2.023 & 2.022 & 2.022 \\
$C_{4}$ & 9.296 & 9.618 & 5.363 & 5.009 & 4.967 & 4.864 & 4.808 & 4.736 & 4.746 & 4.721 & 4.702 & 4.732 & 4.672 & 4.721 \\
$C_{5}$ & 5.600 & 5.632 & 7.301 & 10.827 & 12.496 & 14.399 & 16.801 & 19.500 & 21.715 & 23.915 & 27.051 & 29.188 & 39.921 & 51.309
\end{tabular}

$$
\begin{array}{c|ccccc} 
& C_{1} & C_{2} & C_{3} & C_{4} & C_{5} \\
\mathrm{IM}_{6}= & \mathbf{0} & 0.20 & 0.65 & 0.12 & 0.86 \\
C_{1} & 0.20 & \mathbf{0} & 0.80 & 0.15 & 0.77 \\
C_{3} & 0.65 & 0.80 & \mathbf{0} & 0.66 & 0.35 \\
C_{4} & 0.12 & 0.15 & 0.66 & \mathbf{0} & 0.93 \\
C_{5} & 0.86 & 0.77 & 0.35 & 0.93 & \mathbf{0}
\end{array}
$$

3) Case of best results:

Resulting degrees of "agreement" $\left(\mu_{C, C^{\prime}}\right)$ are as follows:

$$
\begin{array}{c|ccccc} 
& C_{1} & C_{2} & C_{3} & C_{4} & C_{5} \\
\hline \mathrm{IM}_{3}= & \mathbf{1} & 0.74 & 0.49 & 0.63 & 0.36 \\
C_{2} & 0.74 & \mathbf{1} & 0.35 & 0.53 & 0.51 \\
C_{3} & 0.49 & 0.35 & \mathbf{1} & 0.71 & 0.30 \\
C_{4} & 0.63 & 0.53 & 0.71 & \mathbf{1} & 0.25 \\
C_{5} & 0.36 & 0.51 & 0.30 & 0.25 & \mathbf{1}
\end{array}
$$

Resulting degrees of "disagreement" $\left(\nu_{C, C^{\prime}}\right)$ are as follows:

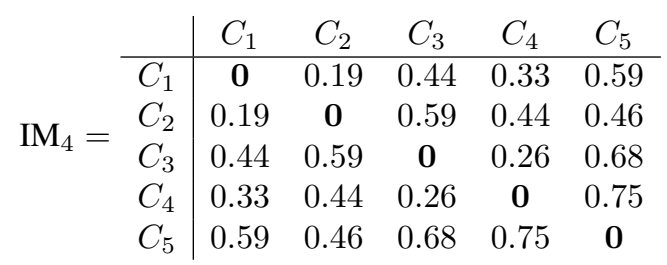

Let us consider the following scheme for defining the consonance and dissonance between each pair of criteria (see Table III).
TABLE II

CRITERIA RELATIONS SORTED By $\mu_{C, C^{\prime}}$ VALUES

\begin{tabular}{|c|c|c|c|}
\hline \multirow{2}{*}{ Criteria relation } & \multicolumn{3}{|c|}{ Obtained $\left\langle\mu_{C, C^{\prime}}, \nu_{C, C^{\prime}}\right\rangle$ values in case of } \\
\cline { 2 - 4 } & average results & worst results & best results \\
\hline$C_{1} \leftrightarrow C_{2}$ & $\langle 0.91,0.08\rangle$ & $\langle 0.79,0.20\rangle$ & $\langle 0.74,0.19\rangle$ \\
\hline$C_{2} \leftrightarrow C_{4}$ & $\langle 0.78,0.21\rangle$ & $\langle 0.84,0.15\rangle$ & $\langle 0.53,0.44\rangle$ \\
\hline$C_{1} \leftrightarrow C_{4}$ & $\langle 0.74,0.26\rangle$ & $\langle 0.88,0.12\rangle$ & $\langle 0.63,0.33\rangle$ \\
\hline$C_{3} \leftrightarrow C_{4}$ & $\langle 0.55,0.44\rangle$ & $\langle 0.33,0.66\rangle$ & $\langle 0.71,0.26\rangle$ \\
\hline$C_{1} \leftrightarrow C_{3}$ & $\langle 0.41,0.58\rangle$ & $\langle 0.34,0.65\rangle$ & $\langle 0.49,0.44\rangle$ \\
\hline$C_{3} \leftrightarrow C_{5}$ & $\langle 0.38,0.60\rangle$ & $\langle 0.64,0.35\rangle$ & $\langle 0.30,0.68\rangle$ \\
\hline$C_{2} \leftrightarrow C_{3}$ & $\langle 0.36,0.62\rangle$ & $\langle 0.18,0.80\rangle$ & $\langle 0.35,0.59\rangle$ \\
\hline$C_{2} \leftrightarrow C_{5}$ & $\langle 0.27,0.71\rangle$ & $\langle 0.22,0.77\rangle$ & $\langle 0.51,0.46\rangle$ \\
\hline$C_{1} \leftrightarrow C_{5}$ & $\langle 0.26,0.74\rangle$ & $\langle 0.14,0.86\rangle$ & $\langle 0.36,0.59\rangle$ \\
\hline$C_{4} \leftrightarrow C_{5}$ & $\langle 0.11,0.89\rangle$ & $\langle 0.07,0.93\rangle$ & $\langle 0.25,0.75\rangle$ \\
\hline
\end{tabular}

In the case of the average values of the examined criteria, in accordance with the scale presented in Table III, we found the following pair dependencies:

- There is no observed strong positive consonance or strong negative consonance between any of the ten criteria pairs. Since the observed values depend on the number of objects if we can expand their number, it is possible to 


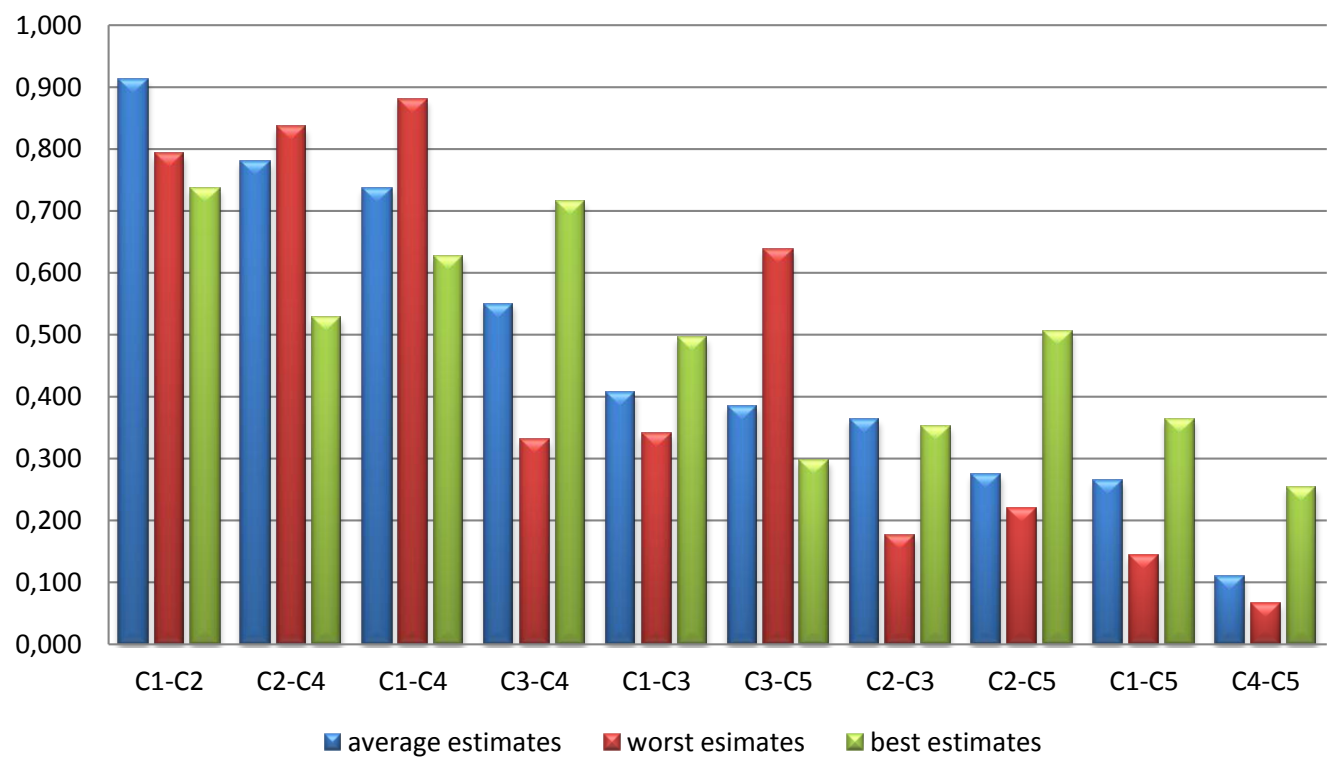

Fig. 1. Degrees of "agreement" $\left(\mu_{C, C^{\prime}}\right.$ values $)$ for all cases

TABLE III

CONSONANCE AND DISSONANCE SCALE

\begin{tabular}{|c|l|}
\hline Interval of $\mu_{C, C^{\prime}}, \%$ & \multicolumn{1}{|c|}{ Meaning } \\
\hline$[0-5]$ & strong negative consonance \\
\hline$(5-15]$ & negative consonance \\
\hline$(15-25]$ & weak negative consonance \\
\hline$(25-33]$ & weak dissonance \\
\hline$(33-43]$ & dissonance \\
\hline$(43-57]$ & strong dissonance \\
\hline$(57-67]$ & dissonance \\
\hline$(67-75]$ & weak dissonance \\
\hline$(75-85]$ & weak positive consonance \\
\hline$(85-95]$ & positive consonance \\
\hline$(95-100]$ & strong positive consonance \\
\hline
\end{tabular}

obtain values in these intervals.

- For the pair $C_{4} \leftrightarrow C_{5}$ (i.e., $T \leftrightarrow J$ ) a negative consonance is identified. Such dependence is logical - for a large number of algorithm iterations (i.e., greater execution time $T$ ) it is more likely to find a more accurate solution, i.e. smaller value of $J$.

- The pairs $C_{2} \leftrightarrow C_{5}$ (i.e., $k_{S} \leftrightarrow T$ ) show identical results - these criteria are in weak dissonance. The third model parameter $Y_{S / X}$ and $T$ are in dissonance. The conclusion is that the total execution time is not dependent solely on one of the model parameters. Logically the triple of these parameters should be in consonance with $T$.
- For the pairs $C_{1} \leftrightarrow C_{3}$ (i.e., $\mu_{\max } \leftrightarrow Y_{S / X}$ ) and $C_{2} \leftrightarrow C_{3}$ (i.e., $k_{S} \leftrightarrow Y_{S / X}$ ) a dissonance is observed. Considering the physical meaning of the model parameters [1] it is clear that there is no dependence between these criteria. A strong correlation is expected between criteria $C_{1} \leftrightarrow C_{2}$ (i.e., $\mu_{\max } \leftrightarrow k_{S}$ ) [1]. The results confirmed these expectation - these criteria are in a positive consonance.

- The observed low value of $\mu_{C_{3}, C_{4}}$, i.e., strong dissonance between $Y_{S / X} \leftrightarrow J$ show the low sensitivity of this model parameter. According to [18] the parameter $Y_{S / X}$ has lower sensitivity compared to parameter $\mu_{\max }$.

- Due to the established strong correlation between criteria $C_{1} \leftrightarrow C_{2}$ (i.e., $\mu_{\max } \leftrightarrow k_{S}$ ) we observe that $C_{1} \leftrightarrow C_{4}$ (i.e., $\mu_{\max } \leftrightarrow J$ ) and $C_{2} \leftrightarrow C_{4}$ (i.e., $k_{S} \leftrightarrow J$ ) are in, respectively weak dissonance and weak positive consonance. Similarly to the relations with $T$ the conclusion is that the accuracy of the criterion is not dependent solely on one of the model parameters. Logically the triple of these parameters should be in consonance (or strong consonance) with the criterion value. Moreover, taking into account the parameters sensitivity it is clear that the more sensitive parameter will be more linked to the value of $J$.

Due to stochastic nature of considered here GA we observed some different criteria dependences in the rest two cases - case of worst and case of best results:

- In the case of the worst results we found weaker relation between $C_{1} \leftrightarrow C_{2}, C_{3} \leftrightarrow C_{4}, C_{2} \leftrightarrow C_{3}, C_{1} \leftrightarrow$ $C_{5}, C_{2} \leftrightarrow C_{5}$ and $C_{4} \leftrightarrow C_{5}$. For the pairs $C_{1} \leftrightarrow$ $C_{4}, C_{2} \leftrightarrow C_{4}$ and $C_{3} \leftrightarrow C_{5}$ we observed higher value of $\mu_{C, C^{\prime}}$. Compared to the case of average results there are no large, strongly manifested discrepancies. In case 
of discrepancy, the considered criteria pair appears in an adjacent scale according to Table III. For example, pair $C_{1} \leftrightarrow C_{2}$ in case of average results are in positive consonance, while in case of worst results - in weak positive consonance.

- In the case of the best results we identify the same results - in case of discrepancy the considered criteria pair appears in an adjacent scale. However, in this case we observed some larger discrepancies. Taking into account the nature of the GA we consider that the results in case of average criteria values have the highest significance.

\section{CONCLUSION}

In this paper based on the apparatus of the Index Matrices and the Intuitionistic Fuzzy Sets, InterCriteria Analysis of a model parameters identification using Genetic Algorithm is performed. A non-linear model of an E. coli fed-batch cultivation process is considered. Series of model identification procedures using Genetic Algorithms are done. The InterCriteria Analysis is applied to explore the existing relations and dependencies of defined model parameters and Genetic Algorithms outcomes - execution time and objective function value. Three case studies are examined - considering average, worst and best results for the obtained model parameters, execution time and objective function value. Applying the InterCriteria Analysis we establish relations and dependencies between the defined criteria. Based on the used scale for defining the consonance and dissonance between each pair of criteria, we discuss which criteria are in consonance and dissonance, as well as the degree of their dependence.

\section{ACKNOWLEDGMENT}

Work presented here is partially supported by the Bulgarian National Scientific Fund under Grant DFNI-I02/5 "InterCriteria Analysis - A New Approach to Decision Making" and by the Polish-Bulgarian collaborative Grant "Parallel and Distributed Computing Practices".

\section{REFERENCES}

[1] G. Bastin and D. Dochain, On-line Estimation and Adaptive Control of Bioreactors, Els. Sc. Publ., 1991.
[2] O. Roeva, T. Pencheva, B. Hitzmann and St. Tzonkov, "A Genetic Algorithms Based Approach for Identification of Escherichia coli Fedbatch Fermentation", Int. J. Bioautomation, Vol. 1, 2004, pp. 30-41.

[3] O. Roeva (Ed.), Real-World Application of Genetic Algorithms, InTech, 2012.

[4] O. Roeva, "Improvement of Genetic Algorithm Performance for Identification of Cultivation Process Models", Advanced Topics on Evolutionary Computing, Book Series: Artificial Intelligence Series - WSEAS, 2008, pp. 34-39.

[5] M. Arndt and B. Hitzmann, "Feed Forward/feedback Control of Glucose Concentration during Cultivation of Escherichia coli", 8th IFAC Int. Conf. on Comp. Appl. in Biotechn, Canada, 2001, pp. 425-429.

[6] D. E. Goldberg, Genetic Algorithms in Search, Optimization and Machine Learning, Addison Wesley Longman, London, 2006.

[7] O. Roeva, S. Fidanova and M. Paprzycki, "Influence of the Population Size on the Genetic Algorithm Performance in Case of Cultivation Process Modelling", Proceedings of the Federated Conference on Computer Science and Information Systems (FedCSIS), WCO 2013, Poland, pp. 371-376.

[8] K. Atanassov, D. Mavrov and V. Atanassova, "Intercriteria Decision Making: A New Approach for Multicriteria Decision Making, Based on Index Matrices and Intuitionistic Fuzzy Sets", Issues in IFSs and GNs, Vol. 11, 2014, pp. 1-8

[9] K. Atanassov, "Generalized index matrices", Comptes Rendus de l'Academie Bulgare des Sciences, Vol. 40, 1987, No. 11, pp. 15-18.

[10] K. Atanassov,“ On index matrices, Part 1: Standard cases”, Advanced Studies in Contemporary Mathematics, Vol. 20, 2010, No. 2, pp. 291 302.

[11] K. Atanassov, "On index matrices, Part 2: Intuitionistic fuzzy case", Proceedings of the Jangjeon Mathematical Society, Vol. 13, 2010, No. 2, pp. 121-126.

[12] K. Atanassov, On Intuitionistic Fuzzy Sets Theory, Springer, Berlin, 2012.

[13] K. Atanassov, E. Szmidt and J. Kacprzyk, "On intuitionistic fuzzy pairs", Notes on Intuitionistic Fuzzy Sets, Vol. 19, 2013, No. 3, pp. 1-13.

[14] V. Atanassova, L. Doukovska, K. Atanassov and D. Mavrov, "InterCriteria Decision Making Approach to EU Member States Competitiveness Analysis", Proc. of the International Symposium on Business Modeling and Software Design - BMSD'14, 24-26 June 2014, Luxembourg, Grand Duchy of Luxembourg, 2014, pp. 289-294.

[15] V. Atanassova, L. Doukovska, D. Mavrov and K. Atanassov, "InterCriteria Decision Making Approach to EU Member States Competitiveness Analysis: Temporal and Threshold Analysis”, P. Angelov et al. (eds.), Intelligent Systems'2014, Advances in Intelligent Systems and Computing, 322, pp. 95-106.

[16] V. Atanassova, D. Mavrov, L. Doukovska and K. Atanassov, "Discussion on the threshold values in the InterCriteria Decision Making approach", Int. J. Notes on Intuitionistic Fuzzy Sets, Volume 20, 2014, Number 2, pp. 94-99.

[17] I. Boussaid, J. Lepagnot and P. Siarry, "A Survey on Optimization Metaheuristics", Information Sciences, Vol. 237, 2013, pp. 82-117.

[18] O. Roeva, "Sensitivity Analysis of E. coli Fed-batch Cultivation Local Models", Mathematica Balkanica, New Series, Vol. 25, 2011, Fasc. 4 pp. 395-411. 